\title{
REGULARITY OF SOLUTIONS OF ABSTRACT QUASILINEAR DELAY INTEGRODIFFERENTIAL EQUATIONS
}

\author{
Dong Gun Park, Krishnan Balachandran, and Francis Paul Samuel
}

\begin{abstract}
We prove the existence and uniqueness of classical solutions for a quasilinear delay integrodifferential equation in Banach spaces. The result is established by using the semigroup theory and the Banach fixed point theorem.
\end{abstract}

\section{Introduction}

Abstract quasilinear integrodifferential equations arise in many areas of science such as population dynamics, mathematical physics, heat conduction theory of material with memory etc. For this reason, this type of equations have received much attention in recent years. The literature related to quasilinear differential and integrodifferential equations is very extensive. A general theory of quasilinear evolution equations has been developed by Kato [14, 15]. Using the method of semigroup, existence and uniqueness of mild and classical solutions of quasilinear evolution equations have been discussed by Pazy [20]. The problem of existence of solutions of quasilinear evolution equations in Banach spaces has been studied by several authors $[2,6,15,16,17,18]$. Pazy [20] considered the following quasilinear equation of the form

$$
\begin{aligned}
u^{\prime}(t)+A(t, u) u(t) & =0, \quad 0<t \leq T, \\
u(0) & =u_{0},
\end{aligned}
$$

and discussed the mild and classical solutions by using the fixed point argument. The existence of classical solution has been studied to the nonhomogeneous quasilinear evolution equation

$$
\begin{aligned}
u^{\prime}(t)+A(t, u) u(t) & =f(t, u), \quad 0<t \leq T, \\
u(0) & =u_{0},
\end{aligned}
$$

Received February 8, 2010; Revised May 14, 2010.

2010 Mathematics Subject Classification. 34G20, 47D03, 47H10.

Key words and phrases. contraction principle, mild and classical solution, semigroup theory.

This Paper is supported by Dong-A University Research Foundation.

(C)2011 The Korean Mathematical Society 
by Furuya [11], Kato [13] and Yagi [21]. Bahuguna [1] proved the existence, uniqueness and continuous dependence of a strong solution of quasilinear integrodifferential equation of the form

$$
\begin{aligned}
u^{\prime}(t)+A(t, u) u(t) & =\int_{0}^{t} a(t-s) k(s, u(s)) d s+f(t), \quad 0 \leq t \leq T \\
u(0) & =u_{0}
\end{aligned}
$$

by using the method of lines (also known as Rothe's method) and the techniques of Crandall and Souganidis [10]. He also established a local classical solution for the same equation in [2]. Oka and Tanaka [19] investigated the existence of classical solution of quasilinear integrodifferential equation of the hyperbolic type

$$
\begin{aligned}
u^{\prime}(t) & =A(t, u) u(t)+\int_{0}^{t} \mathcal{F}(t, s, u(s)) u(s) d s, \quad 0 \leq t \leq T, \\
u(0) & =u_{0},
\end{aligned}
$$

in a pair of Banach spaces $(Y, X)$ such that $Y$ is continuously imbedded in $X$. Oka [18] proved the existence of classical solution of abstract quasilinear integrodifferential equations. Balachandran and Uchiyama [5] discussed the existence and uniqueness of local mild and classical solutions of quasilinear integrodifferential equations. Recently Balachandran and Park [3] studied the existence of solutions of quasilinear integrodifferential evolution equations by using the Schauder fixed point theorem.

In this paper we study the following quasilinear delay integrodifferential equation with time varying delay of the form

$$
\begin{aligned}
& u^{\prime}(t)+A(t, u) u(t) \\
&= K(u)(t)+f\left(t, u(t), u\left(\beta(t), \int_{0}^{t} b(t-s) h(s, u(s), u(\gamma(s))) d s\right),\right. \\
& u(0)=u_{0}, t \in[0, T]=I,
\end{aligned}
$$

where $A(t, u)$ is the infinitesimal generator of a $C_{0}$-semigroup in a Banach space $X, u_{0} \in X, f: I \times X \times X \times X \rightarrow X, h: I \times X \times X \rightarrow X$ are functions and $K$ is the nonlinear Volterra operator

$$
K(u)(t)=\int_{0}^{t} a(t-s) k(s, u(s), u(\alpha(s))) d s .
$$

Here $a, b: I \rightarrow I$ are real-valued continuous functions, $k: I \times X \times X \rightarrow X$, $\alpha, \beta, \gamma: I \rightarrow I$ are absolutely continuous functions. The equations considered in $[1,11,13,20,21]$ are particular cases of the equation (1)-(2) and generalize the results of $[3,4,12,14,15,20]$. 


\section{Preliminaries}

Let $X$ and $Y$ be two Banach spaces such that $Y$ is densely and continuously embedded in $X$. For any Banach space $Z$, the norm of $Z$ is denoted by $\|\cdot\|$ or $\|\cdot\|_{Z}$. The space of all bounded linear operators from $X$ to $Y$ is denoted by $B(X, Y)$ and $B(X, X)$ is written as $B(X)$. We recall some definitions and known facts from Pazy [20].

Definition 2.1. Let $S$ be a linear operator in $X$ and let $Y$ be a subspace of $X$. The operator $\tilde{S}$ defined by $D(\tilde{S})=\{x \in D(S) \cap Y: S x \in Y\}$ and $\tilde{S} x=S x$ for $x \in D(\tilde{S})$ is called the part of $S$ in $Y$.

Definition 2.2. Let $B$ be a subset of $X$ and, for every $0 \leq t \leq T$ and $b \in B$, let $A(t, b)$ be the infinitesimal generator of a $C_{0}$ semigroup $\bar{S}_{t, b}(s), s \geq 0$, on $X$. The family of operators $\{A(t, b),(t, b) \in I \times B\}$, is stable if there are constants $M \geq 1$ and $\omega$ such that

$$
\begin{aligned}
& \rho(A(t, b)) \supset(\omega, \infty) \text { for }(t, b) \in I \times B, \\
& \left\|\prod_{j=1}^{k} R\left(\lambda: A\left(t_{j}, b_{j}\right)\right)\right\| \leq M(\lambda-\omega)^{-k}
\end{aligned}
$$

for $\lambda>\omega, 0 \leq t_{1} \leq t_{2} \leq \cdots \leq t_{k} \leq T, b_{j} \in B, 1 \leq j \leq k$.

The stability of $\{A(t, b)\},(t, b) \in I \times B$ implies (see [20]) that

$$
\left\|\prod_{j=1}^{k} S_{t_{j}, b_{j}}\left(s_{j}\right)\right\| \leq M \exp \left\{\omega \sum_{j=1}^{k} s_{j}\right\}, \quad s_{j} \geq 0
$$

$0 \leq t_{1} \leq t_{2} \leq \cdots \leq t_{k} \leq T, \quad b_{j} \in B, 1 \leq j \leq k . \quad k=1,2, \ldots$

Definition 2.3. Let $S_{t, b}(s), s \geq 0$, be the $C_{0}$-semigroup generated by $A(t, b)$, $(t, b) \in I \times B$. A subspace $Y$ of $X$ is called $A(t, b)$-admissible if $Y$ is an invariant subspace of $S_{t, b}(s)$ and the restriction of $S_{t, b}(s)$ to $Y$ is a $C_{0}$-semigroup in $Y$.

Let $B \subset X$ be a subset of $X$ such that, for every $(t, b) \in I \times B, A(t, b)$ is the infinitesimal generator of a $C_{0}$-semigroup $S_{t, b}(s), s \geq 0$ on $X$. We make the following assumptions:

(H1) The family $\{A(t, b)\},(t, b) \in I \times B$, is stable.

(H2) $Y$ is $A(t, b)$-admissible for $(t, b) \in I \times B$ and the family $\{\tilde{A}(t, b)\},(t, b) \in$ $I \times B$ of parts $\tilde{A}(t, b)$ of $A(t, b)$ in $Y$ is stable in $Y$.

(H3) For $(t, b) \in I \times B, D(A(t, b)) \supset Y, A(t, b)$ is a bounded linear operator from $Y$ to $X$ and $t \rightarrow A(t, b)$ is continuous in the $B(Y, X)$ norm $\|\cdot\|$ for every $b \in B$.

(H4) There is a positive constant $N$ such that

$$
\left\|A\left(t, b_{1}\right)-A\left(t, b_{2}\right)\right\|_{Y \rightarrow X} \leq N\left\|b_{1}-b_{2}\right\|_{X}
$$

holds for every $b_{1}, b_{2} \in B$ and $0 \leq t \leq T$. 
Let $B$ be a subset of $X$ and $\{A(t, b),(t, b) \in I \times B\}$, be a family of operators satisfying the conditions (H1)-(H4). If $u \in C(I: X)$ has values in $B$, then there is a unique evolution system $U_{u}(t, s), 0 \leq s \leq t \leq T$, in $X$ satisfying (see Theorem 5.3.1 and Lemma 6.4.2 in [20] pp. 135, 201-202).

(i) $\left\|U_{u}(t, s)\right\| \leq K_{1} e^{\omega(t-s)} \quad$ and $\quad 0 \leq s \leq t \leq T$, where $K_{1}$ and $\omega$ are stability constants.

(ii) $\frac{\partial^{+}}{\partial t} U_{u}(t, s) y=A(s, u(s)) U_{u}(t, s) y$ for $y \in Y$, and $0 \leq s \leq t \leq T$.

(iii) $\frac{\partial}{\partial s} U_{u}(t, s) y=-U_{u}(t, s) A(s, u(s)) y$ for $y \in Y$, and $0 \leq s \leq t \leq T$.

(H5) For every $u \in C(I: X)$ satisfying $u(t) \in B$ for $0 \leq t \leq T$, we have

$$
U_{u}(t, s) Y \subset Y, \quad 0 \leq s \leq t \leq T,
$$

where $U_{u}(t, s)$ is strongly continuous in $Y$ for $0 \leq s \leq t \leq T$.

(H6) $X$ and $Y$ are reflexive Banach spaces and there exist an isometry between them.

(H7) For every $\left(t, b_{1}, b_{2}, b_{3}\right) \in I \times B \times B, f\left(t, b_{1}, b_{2}, b_{3}\right) \in Y$.

(H8) The real-valued function $a$ and $b$ are continuous on $I$ and there exist positive constants $a_{T}$ and $b_{T}$ such that $|a(t)| \leq a_{T}$ and $|b(t)| \leq b_{T}$ for $t \in I$.

(H9) $\alpha, \beta, \gamma: I \rightarrow I$ are absolutely continuous and there exist constants $\delta_{i}>0, \quad i=1,2,3$ such that $\alpha^{\prime}(t) \geq \delta_{1}, \beta^{\prime}(t) \geq \delta_{2}$ and $\gamma^{\prime}(t) \geq \delta_{3}$ respectively for $0<t \leq T$.

(H10) The nonlinear map $k: I \times X \times X \rightarrow X$ satisfies

$\int_{0}^{t}\left\|k\left(s, x_{1}, y_{1}\right)-k\left(s, x_{2}, y_{2}\right)\right\| d s \leq N_{A}\left[\left\|x_{1}(t)-x_{2}(t)\right\|+\left\|y_{1}(t)-y_{2}(t)\right\|\right]$ for a.e. $t \in I$, where $N_{A}$ and $N_{0}$ are positive constants and

$$
N_{0}=\max \int_{0}^{t}\|k(s, 0,0)\| d s .
$$

For the conditions (H11) and (H12), let $Z$ be taken as both $X$ and $Y$

(H11) $f: I \times Z \times Z \times Z \rightarrow Z$ is continuous and there exist constants $F_{A}>0$ and $F_{0}>0$ such that

$$
\begin{aligned}
& \left\|f\left(t, u_{1}, v_{1}, w_{1}\right)-f\left(t, u_{2}, v_{2}, w_{2}\right)\right\|_{Z} \\
\leq & F_{A}\left(\left\|u_{1}-u_{2}\right\|_{Z}+\left\|v_{1}-v_{2}\right\|_{Z}+\left\|w_{1}-w_{2}\right\|_{Z}\right), \\
F_{0}= & \max _{\tau \in I}\|f(t, 0,0,0)\|_{Z} .
\end{aligned}
$$

(H12) $h: I \times Z \times Z \rightarrow Z$ is continuous and there exist constants $H_{A}>0$ and $H_{0}>0$ such that

$\int_{0}^{t}\left\|h\left(s, u_{1}, v_{1}\right)-h\left(s, u_{2}, v_{2}\right)\right\|_{Z} d s \leq H_{A}\left(\left\|u_{1}(t)-u_{2}(t)\right\|_{Z}+\left\|v_{1}(t)-v_{2}(t)\right\|\right)$, 


$$
H_{0}=\max \left\{\int_{0}^{t}\|h(s, 0,0)\|_{Z} d s\right\} .
$$

Further, there exist a positive constant $K_{0}$ such that for every $u, v \in C(I ; X)$ with values in $B$ and every $y \in Y$, we have

$$
\left\|U_{u}(t, s) y-U_{v}(t, s) y\right\|_{X} \leq K_{0}\|y\|_{Y} \int_{s}^{t}\|u(\tau)-v(\tau)\|_{X} d \tau .
$$

For details of the above mentioned results, we refer to Theorem 6.4 .3 and Lemma 6.4.4 in Pazy [20].

To prove our main result we need the following theorems.

Theorem 2.1 (Theorem 5.5.2 [20]). Let $A(t), 0 \leq t \leq T$, be the infinitesimal generator of a $C_{0}$ semigroup $S_{t}(s), s \leq 0$ on $X$. If the family satisfying the conditions $\left(\mathrm{H}_{1}\right)-\left(\mathrm{H}_{3}\right)[20$, Page 135$]$, then there exists a unique evolution system $U(t, s), 0 \leq t \leq s \leq T$, in $X$ satisfying

(E1) $\|U(t, s)\| \leq M e^{\omega(t-s)} \quad$ and $\quad 0 \leq s \leq t \leq T$,

(E2) $\left.\frac{\partial^{+}}{\partial t} U(t, s) v\right|_{t=s}=A(s) v$ for $v \in Y$, and $0 \leq s \leq T$,

(E3) $\frac{\partial}{\partial s} U(t, s) v=-U(t, s) A(s) v \quad$ for $v \in Y$, and $0 \leq s \leq t \leq T$,

where the derivative from the right in (E2) and the derivative in (E3) are in the strong sense in $X$.

Theorem 2.2 (Theorem 5.5.2[20]). Let $A(t)_{t \in[0, T]}$ satisfy the conditions of Theorem 2.1 and let $U(t, s), 0 \leq s \leq t \leq T$ be the evolution system given in Theorem 2.1. If

(E4) $U(t, s) Y \supset Y$ for $0 \leq s \leq t \leq T$.

(E5) For $v \in Y, U(t, s) v$ is continuous in $Y$ for $0 \leq s \leq t \leq T$, then for every $v \in Y, U(t, s) v$ is the unique $Y$-valued solution of the initial value problem

$$
\begin{aligned}
\frac{d u(t)}{d t} & =A(t) u(t) \text { for } 0 \leq s<t \leq T, \\
u(s) & =v .
\end{aligned}
$$

Theorem 2.3 (Theorem 5.5.2[20]). Let $\{A(t)\}_{t \in[0, T]}$ satisfy the condition of Theorem 2.2. If $f \in C([s, T]: Y)$, then for every $v \in Y$ the initial value problem

$$
\begin{aligned}
\frac{d u(t)}{d t} & =A(t) u(t)+f(t) \text { for } 0 \leq s<t \leq T, \\
u(s) & =v
\end{aligned}
$$

possesses a unique $Y$-valued solution u given by

$$
u(t)=U(t, s) v+\int_{s}^{t} U(t, \eta) f(\eta) d \eta .
$$




\section{Existence of solutions}

By a mild solution of (1)-(2), we mean a function $u \in C(I: X)$ and $u_{0} \in X$ satisfying the integral equation

$$
\begin{aligned}
u(t)= & U_{u}(t, 0) u_{0}+\int_{0}^{t} U_{u}(t, s)[K(u)(s) \\
& \left.+f\left(s, u(s), u(\beta(s)), \int_{0}^{s} b(s-\tau) h(\tau, u(\tau), u(\gamma(\tau))) d \tau\right)\right] d s .
\end{aligned}
$$

A function $u \in C(I: X)$ such that $u(t) \in D(A(t, u(t)))$ for $t \in(0, T], u \in$ $C^{1}((0, T]: X)$ and satisfies (1)-(2) in $X$ is called a classical solution of (1)-(2) on $[0, T]$.

Theorem 3.1. Let $u_{0} \in Y$ and the family $A(t, b)$ of linear operators for $t \in$ $I=[0, T]$ and $b \in B=\left\{u \in Y:\|u\|_{Y} \leq r\right\}, r>0$, satisfy the assumptions (H1)-(H12) and $A(t, b) u_{0} \in Y$ with

$$
\left\|A(t, b) u_{0}\right\|_{Y} \leq C_{A}, C_{A}>0
$$

for all $(t, b) \in I \times B$. Then there is a positive constant $T_{0}$ such that the quasilinear problem (1)-(2) has a unique classical solution $u \in C\left(\left[0, T_{0}\right]: Y\right) \cap$ $C^{1}\left(\left(0, T_{0}\right]: X\right)$.

Proof. First we prove the existence of a unique local mild solution for (1) and (2). The assumption (H5) implies that there exists a constant $K_{1}>0$ such that

$$
\left\|U_{u}(t, s)\right\|_{B(Y)} \leq K_{1}
$$

for $s \leq t, s, t \in I$ and every $u \in C(I: X)$. Choose

$$
T_{0}=\min \left\{T, \frac{1}{2 K_{1} C_{A}}, \frac{1}{2 T_{1}}, \frac{1}{2 T_{2}}\right\},
$$

where

$$
\begin{aligned}
T_{1}=K_{1}\left\{r\left[a_{T} N_{A}\left(1+1 / \delta_{1}\right)+F_{A}\left(1+1 / \delta_{2}\right)+b_{T} F_{A} H_{A}\left(1+1 / \delta_{3}\right)\right]\right. \\
\left.+a_{T} N_{0}+b_{T} F_{A} H_{0}+F_{0}\right\}
\end{aligned}
$$

and

$$
\begin{aligned}
T_{2}= & K_{0}\left\|u_{0}\right\|_{Y}+K_{0} T\left[r\left[a_{T} N_{A}\left(1+1 / \delta_{1}\right)+F_{A}\left(1+1 / \delta_{2}\right)+b_{T} F_{A} H_{A}\left(1+1 / \delta_{3}\right)\right]\right. \\
& \left.+a_{T} N_{0}+b_{T} F_{A} H_{0}+F_{0}\right]+K_{1}\left[a_{T} N_{A}\left(1+1 / \delta_{1}\right)+F_{A}\left(1+1 / \delta_{2}\right)\right. \\
& \left.+b_{T} F_{A} H_{A}\left(1+1 / \delta_{3}\right)\right] .
\end{aligned}
$$

Let $S$ be the subset of $C\left(\left[0, T_{0}\right]: X\right)$ defined by

$$
S=\left\{u: u \in C\left(\left[0, T_{0}\right]: X\right),\|u(t)\|_{Y} \leq r, u(0)=u_{0} \text { for } 0 \leq t \leq T_{0}\right\} .
$$


Define a mapping $P: S \rightarrow S$ given by

$$
\begin{aligned}
(P u)(t)= & U_{u}(t, 0) u_{0}+\int_{0}^{t} U_{u}(t, s)[K(u)(s) \\
& \left.+f\left(s, u(s), u(\beta(s)), \int_{0}^{s} b(s-\tau) h(\tau, u(\tau), u(\gamma(\tau))) d \tau\right)\right] d s .
\end{aligned}
$$

We claim that $P$ maps $S$ into $S$. Clearly $P u(0)=u_{0}$ and $P u(t) \in Y$ for $0 \leq t \leq T_{0}$ and it remains to show that $\|P u(t)\| \leq r$ in $Y$. Integrating (iii) in $X$ from 0 to $t$, we find

and hence

$$
U_{u}(t, 0) u_{0}-u_{0}=-\int_{0}^{t} U_{u}(t, \tau) A(\tau, u(\tau)) u_{0} d \tau
$$

$$
\left\|U_{u}(t, 0) u_{0}-u_{0}\right\| \leq K_{1} C_{A} T_{0}
$$

Also we have

$$
\begin{aligned}
& \|P u(t)\| \\
= & \left\|U_{u}(t, 0) u_{0}-u_{0}\right\|+\int_{0}^{t} \| U_{u}(t, s)\left[\int_{0}^{s} a(s-\tau) k(\tau, u(\tau), u(\alpha(\tau))) d \tau\right. \\
& \left.+f\left(s, u(s), u(\beta(s)), \int_{0}^{s} b(s-\tau) h(\tau, u(\tau) u(\gamma(\tau))) d \tau\right)\right] \| d s \\
\leq & K_{1} C_{A} T_{0}+K_{1}\left\{\int_{0}^{t} \int_{0}^{s}\|a(s-\tau)\|[\|k(\tau, u(\tau), u(\alpha(s)))-k(\tau, 0,0)\|\right. \\
& +\|k(\tau, 0,0)\|] d \tau d s+\int_{0}^{t}\left[\| f\left(s, u(s), u(\beta(s)), \int_{0}^{s} b(s-\tau) h(\tau, u(\tau), u(\gamma(\tau))) d \tau\right)\right. \\
& -f(s, 0,0,0)\|+\| f(s, 0,0,0) \|] d s\} .
\end{aligned}
$$

Using the assumptions (H8)-(H12), we get

$$
\begin{aligned}
& \|P u(t)\| \\
\leq & K_{1}\left\{C_{A} T_{0}+a_{T} N_{A} \int_{0}^{t}(\|u(s)\|+\|u(\alpha(s))\|) d s+a_{T} N_{0} T_{0}\right. \\
& +F_{A} \int_{0}^{t}\left(\|u(s)\|+\|u(\beta(s))\|+\| b(s-\tau) h(\tau, u(\tau), u(\gamma(\tau)) d \tau \|) d s+F_{0} T_{0}\right\} \\
\leq & K_{1}\left\{C_{A} T_{0}+a_{T} N_{A} r T_{0}+a_{T} N_{A} / \delta_{1} \int_{\alpha(0)}^{\alpha(t)}\|u(s)\| d s+a_{T} N_{0} T_{0}+F_{A} r T_{0}\right. \\
& +F_{A} / \delta_{2} \int_{\beta(0)}^{\beta(t)}\|u(s)\| d s+b_{T} F_{A} H_{A} r T_{0}+b_{T} F_{A} H_{A} / \delta_{3} \int_{\gamma(0)}^{\gamma(t)}\|u(s)\| d s \\
& \left.+b_{T} F_{A} H_{0} T_{0}+F_{0} T_{0}\right\} \\
\leq & K_{1} T_{0}\left\{C_{A}+r\left[a_{T} N_{A}\left(1+1 / \delta_{1}\right)+F_{A}\left(1+1 / \delta_{2}\right)+b_{T} F_{A} H_{A}\left(1+1 / \delta_{3}\right)\right]\right. \\
& \left.+a_{T} N_{0}+b_{T} F_{A} H_{0}+F_{0}\right\} .
\end{aligned}
$$


From the assumption, we get $\|P u(t)\| \leq r$. Therefore $P$ maps $S$ into itself. Moreover, if $u, v \in S$, then

$$
\begin{aligned}
& \|P u(t)-P v(t)\| \\
\leq & \left\|U_{u}(t, 0) u_{0}-U_{v}(t, 0) u_{0}\right\| \\
& +\int_{0}^{t} \| U_{u}(t, s)\left[K(u)(s)+f\left(s, u(s), u(\beta(s)), \int_{0}^{s} b(s-\tau) h(\tau, u(\tau), u(\gamma(\tau))) d \tau\right)\right] \\
& -U_{v}(t, s)\left[K(v)(s)+f\left(s, v(s), v(\beta(s)), \int_{0}^{s} b(s-\tau) h(\tau, v(\tau), v(\gamma(\tau))) d \tau\right)\right] \| d s \\
\leq & \left\|U_{u}(t, 0) u_{0}-U_{v}(t, 0) u_{0}\right\| \\
& +\int_{0}^{t} \| U_{u}(t, s)\left[K(u)(s)+f\left(s, u(s), u(\beta(s)), \int_{0}^{s} b(s-\tau) h(\tau, u(\tau), u(\gamma(\tau))) d \tau\right)\right] \\
& -U_{v}(t, s)\left[K(u)(s)+f\left(s, u(s), u(\beta(s)), \int_{0}^{s} b(s-\tau) h(\tau, u(\tau), u(\gamma(\tau))) d \tau\right)\right] \| d s \\
& -U_{v}(t, s)\left[K(v)(s)+f\left(s, v(s), v(\beta(s)), \int_{0}^{s} b(s-\tau) h(\tau, v(\tau), v(\gamma(\tau))) d \tau\right)\right] \| d s .
\end{aligned}
$$

From our assumption, we have

$$
\begin{aligned}
I_{1} \leq & K_{0}\left\|u_{0}\right\|_{Y} T_{0} \max _{\tau \in I}\|u(\tau)-v(\tau)\|, \\
I_{2} \leq & \int_{0}^{t}\left\|U_{u}(t, s)-U_{v}(t, s)\right\|[\|K(u)(s)\| \\
& \left.+\left\|f\left(s, u(s), u(\beta(s)), \int_{0}^{s} b(s-\tau) h(\tau, u(\tau), u(\gamma(\tau))) d \tau\right)\right\|\right] d s \\
\leq & K_{0} T T_{0}\left\{r\left[a_{T} N_{A}\left(1+1 / \delta_{1}\right)+F_{A}\left(1+1 / \delta_{2}\right)+b_{T} F_{A} H_{A}\left(1+1 / \delta_{3}\right)\right]\right. \\
& \left.+a_{T} N_{0}+b_{T} F_{A} H_{0}+F_{0}\right\} \max _{\tau \in I}\|u(\tau)-v(\tau)\|, \\
I_{3} \leq & \int_{0}^{t}\left\|U_{v}(t, s)\right\|[\|K(u)(s)-K(v)(s)\| \\
& +\| f\left(s, u(s), u(\beta(s)), \int_{0}^{s} b(s-\tau) h(\tau, u(\tau), u(\gamma(\tau))) d \tau\right) \\
& \left.\quad-f\left(s, v(s), v(\beta(s)), \int_{0}^{s} b(s-\tau) h(\tau, v(\tau), v(\gamma(\tau))) d \tau\right) \|\right] d s \\
\leq & K_{1} T_{0}\left\{a_{T} N_{A}\left(1+1 / \delta_{1}\right)+F_{A}\left(1+1 / \delta_{2}\right)+b_{T} F_{A} H_{A}\left(1+1 / \delta_{3}\right)\right\} \\
& \quad \times \max _{\tau \in I}\|u(\tau)-v(\tau)\| .
\end{aligned}
$$

From these inequalities it follows that, for any $t \in I$,

$$
\|P u(t)-P v(t)\| \leq \frac{1}{2} \max _{\tau \in I}\|u(\tau)-v(\tau)\|,
$$


so that $P$ is a contraction on $S$. From the contraction mapping theorem, it follows that $P$ has a unique fixed point $u \in S$ which is the mild solution of (1) and $(2)$ on $\left[0, T_{0}\right]$.

Now we consider the evolution equation

$$
\begin{aligned}
v^{\prime}(t)+B(t) v(t) & =l(t), \quad t \in\left[0, T_{0}\right], \\
v(0) & =u_{0},
\end{aligned}
$$

where $B(t)=A(t, u(t))$ and $l(t)=K(u)(t)+f\left(t, u(t), u(\beta(t)), \int_{0}^{t} b(t-s) h(s\right.$, $u(s), u(\gamma(s)) d s, t \in\left[0, T_{0}\right]$, and $u$ is the unique fixed point of $P$ in $S$. We note that $B(t)$ satisfies $\left(\mathrm{H}_{1}\right)-\left(\mathrm{H}_{3}\right)$ of $[20]$ (Section 5.5.3) and $l \in C(I: Y)$. Theorem 5.5.2 in [20] implies that there exists a unique function $v \in C(I: Y)$ such that $v \in C^{1}\left(\left(0, T_{0}\right]: X\right)$ satisfying $(1)$ and $(2)$ in $X$ and $v$ is given by

$$
\begin{aligned}
v(t)= & U_{u}(t, 0) u_{0}+\int_{0}^{t} U_{u}(t, s)[K(u)(s) \\
& +f\left(s, u(s), u(\beta(s)), \int_{0}^{s} b(s-\tau)(\tau, u(\tau), u(\gamma(\tau))) d \tau\right] d s,
\end{aligned}
$$

where $U_{u}(t, s)$ is the evolution system generated by the family $\{A(t, u(t))\}, t \in$ $I$, of the linear operators in $X$. The uniqueness of $v$ implies that $v=u$ on $t \in\left[0, T_{0}\right]$. Hence $u$ is a unique local classical solution of (1)-(2) and $u \in$ $C\left(\left[0, T_{0}\right]: Y\right) \cap C^{1}\left(\left(0, T_{0}\right]: X\right)$.

\section{Nonlocal Cauchy problem}

The nonlocal Cauchy problem for semilinear evolution equations in Banach space was studied first by Byszewski $[7,8,9]$ where he established the existence and uniqueness of mild and classical solutions. The nonlocal conditions were motivated by physical problems and their importance is discussed in $[7,8,9]$. Balachandran et al $[3,4,5,6,12]$ studied the nonlocal Cauchy problem for various type of quasilinear integrodifferential equations. Consider the nonlocal condition of the form

$$
u(0)+g(u)=u_{0}, t \in[0, T]=I
$$

for the quasilinear integrodifferential equation (1).

Assume the following conditions:

(H13) $g: C(I: B) \rightarrow Y$ is Lipschitz continuous in $X$ and bounded in $Y$, that is, there exist constants $G>0$ and $G_{1}>0$ such that

$$
\begin{aligned}
\|g(u)\|_{Y} & \leq G \\
\|g(u)-g(v)\|_{Y} & \leq G_{1} \max _{t \in I}\|u(t)-v(t)\|_{X} .
\end{aligned}
$$

(H14) There exists a positive constant $r>0$ such that

$$
K_{1}\left\{\left\|u_{0}\right\|_{Y}+G+T_{0}\left[r \left[a_{T} N_{A}\left(1+1 / \delta_{1}\right)\right.\right.\right.
$$




$$
\begin{aligned}
& \left.\left.\left.+F_{A}\left(1+1 / \delta_{2}\right)+b_{T} F_{A} H_{A}\left(1+1 / \delta_{3}\right)\right]+a_{T} N_{0}+b_{T} F_{A} H_{0}+F_{0}\right]\right\} \leq r, \\
q=\{ & K_{0} T_{0}\left[\left\|u_{0}\right\|_{Y}+G\right]+K_{1} G_{1}+K_{0} T T_{0}\left[r \left[a_{T} N_{A}\left(1+1 / \delta_{1}\right)+F_{A}\left(1+1 / \delta_{2}\right)\right.\right. \\
& \left.\left.+b_{T} F_{A} H_{A}\left(1+1 / \delta_{3}\right)\right]+a_{T} N_{0}+b_{T} F_{A} H_{0}+F_{0}\right]+K_{1} T_{0}\left[a_{T} N_{A}\left(1+1 / \delta_{1}\right)\right. \\
& \left.\left.+F_{A}\left(1+1 / \delta_{2}\right)+b_{T} F_{A} H_{A}\left(1+1 / \delta_{3}\right)\right]\right\}<1 .
\end{aligned}
$$

By a mild solution of (1) and (11), we mean a function $u \in C(I: X)$ and $u_{0} \in X$ satisfying the integral equation

$$
\begin{aligned}
u(t)= & U_{u}(t, 0) u_{0}-U_{u}(t, 0) g(u)+\int_{0}^{t} U_{u}(t, s)[K(u)(s) \\
& \left.+f\left(s, u(s), u(\beta(s)), \int_{0}^{s} b(t-s) h(\tau, u(\tau), u(\gamma(\tau))) d \tau\right) d s\right] .
\end{aligned}
$$

A function $u \in C(I ; X)$ such that $u(t) \in D(A(t, u(t)))$ for $t \in(0, T], u \in$ $C^{1}((0, T]: X)$ and satisfies (1) and (11) in $X$ is called a classical solution of (1) and $(11)$ on $[0, T]$.

Theorem 4.1. Let $u_{0} \in Y$ and $B=\left\{u \in Y:\|u\|_{Y} \leq r\right\}, r>0$. If the assumptions (H1)-(H14) are satisfied, then there is a positive constant $T_{0}$ such that the quasilinear problem (1) and (11) has a unique classical solution $u \in$ $C\left(\left[0, T_{0}\right]: Y\right) \cap C^{1}\left(\left(0, T_{0}\right]: X\right)$.

Proof. First we prove the existence of a unique mild solution for (1) and (11). The assumption (H5) implies that there exists a constant $K_{1}>0$ such that

$$
\left\|U_{u}(t, s)\right\|_{B(Y)} \leq K_{1}
$$

for $s \leq t, s, t \in I$ and every $u \in C(I ; X)$. Let $S$ be the subset of $C\left(\left[0, T_{0}\right]: X\right)$ defined by

$$
S=\left\{u: u \in C\left(\left[0, T_{0}\right]: X\right),\|u(t)\| \leq r \text { for } 0 \leq t \leq T_{0}\right\} .
$$

Define a mapping $Q: S \rightarrow S$ by

$$
\begin{aligned}
Q u(t)= & U_{u}(t, 0) u_{0}-U_{u}(t, 0) g(u)+\int_{0}^{t} U_{u}(t, s)[K(u)(s) \\
& \left.+f\left(s, u(s), u(\beta(s)), \int_{0}^{s} b(t-s) h(\tau, u(\tau), u(\gamma(\tau))) d \tau\right)\right] d s .
\end{aligned}
$$

We claim that $Q$ maps $S$ into $S$. For $u \in S$, we have

$$
\begin{aligned}
& \|Q u(t)\| \\
= & \left\|U_{u}(t, 0) u_{0}-U_{u}(t, 0) g(u)\right\| \\
& +\int_{0}^{t} \| U_{u}(t, s)\left[K(u)(s)+f\left(s, u(s), u(\beta(s)), \int_{0}^{s} b(t-s) h(\tau, u(\tau) u(\gamma(\tau))) d \tau\right] d s \|\right. \\
\leq & K_{1}\left\{\left\|u_{0}\right\|_{Y}+G+T_{0}\left[r\left[a_{T} N_{A}\left(1+1 / \delta_{1}\right)+F_{A}\left(1+1 / \delta_{2}\right)+b_{T} F_{A} H_{A}\left(1+1 / \delta_{3}\right)\right]\right.\right.
\end{aligned}
$$




$$
\left.\left.+a_{T} N_{0}+b_{T} F_{A} H_{0}+F_{0}\right]\right\} .
$$

From the assumption (H14), one gets $\|Q u(t)\| \leq r$. Therefore $Q$ maps $S$ into itself. Moreover, if $u, v \in S$, then

$$
\begin{aligned}
& \|Q u(t)-Q v(t)\| \\
\leq & \left\|U_{u}(t, 0) u_{0}-U_{v}(t, 0) u_{0}\right\|+\left\|U_{u}(t, 0) g(u)-U_{v}(t, 0) g(v)\right\| \\
& +\int_{0}^{t} \| U_{u}(t, s)\left[K(u)(s)+f\left(s, u(s), u(\beta(s)), \int_{0}^{s} b(t-s) h(\tau, u(\tau), u(\gamma(\tau))) d \tau\right)\right] \\
& -U_{v}(t, s)\left[K(v)(s)+f\left(s, v(s), v(\beta(s)), \int_{0}^{s} b(t-s) h(\tau, v(\tau), v((\gamma(\tau))) d \tau)\right] \| d s\right. \\
\leq & \left\{K_{0} T_{0}\left[\left\|u_{0}\right\|_{Y}+G\right]+K_{1} G_{1}+K_{0} T T_{0}\left[r \left[a_{T} N_{A}\left(1+1 / \delta_{1}\right)+F_{A}\left(1+1 / \delta_{2}\right)\right.\right.\right. \\
& \left.\left.+b_{T} F_{A} H_{A}\left(1+1 / \delta_{3}\right)\right]+a_{T} N_{0}+b_{T} F_{A} H_{0}+F_{0}\right]+K_{1} T_{0}\left[a_{T} N_{A}\left(1+1 / \delta_{1}\right)\right. \\
& \left.\left.+F_{A}\left(1+1 / \delta_{2}\right)+b_{T} F_{A} H_{A}\left(1+1 / \delta_{3}\right)\right]\right\} \max _{\tau \in I}\|u(\tau)-v(\tau)\| \\
= & q \max _{\tau \in I}\|u(\tau)-v(\tau)\|,
\end{aligned}
$$

where $0<q<1$. From these inequalities, it follows that, for any $t \in I$,

$$
\|Q u(t)-Q v(t)\| \leq q \max _{\tau \in I}\|u(\tau)-v(\tau)\|,
$$

so that $Q$ is a contraction on $S$. Hence $Q$ has a unique fixed point $u \in S$ such that $Q u(t)=u(t)$ which is the mild solution of (1) and (11).

Now we consider the evolution equation

$$
\begin{aligned}
w^{\prime}(t)+B(t) w(t) & =v(t), \quad t \in\left[0, T_{0}\right], \\
w(0) & =u_{0}-g(u),
\end{aligned}
$$

where $B(t)=A(t, u(t))$ and $v(t)=K(u)(t)+f\left(t, u(t), u(\beta(t)), \int_{0}^{t} b(t-s) h(s\right.$, $u(s), u(\gamma(s)) d s, t \in\left[0, T_{0}\right]$ and $u$ is the unique fixed point of $Q$ in $S$. We note that $B(t)$ satisfies $\left(\mathrm{H}_{1}\right)-\left(\mathrm{H}_{3}\right)$ of [20] (Section 5.5.3) and $v \in C(I: Y)$. Theorem 5.5.2 in [20] implies that there exists a unique function $w \in C(I: Y)$ such that $w \in C^{1}\left(\left(0, T_{0}\right]: X\right)$ satisfying (1) and (11) in $X$ and $w$ is given by

$$
\begin{aligned}
w(t)= & U_{u}(t, 0) u_{0}-U_{u}(t, 0) g(u)+\int_{0}^{t} U_{u}(t, s)[K(u)(s) \\
& +f\left(s, u(s), u(\beta(s)), \int_{0}^{s} b(s-\tau)(\tau, u(\tau), u(\gamma(\tau))) d \tau\right] d s,
\end{aligned}
$$

where $U_{u}(t, s)$ is the evolution system generated by the family $\{A(t, u(t))\}, t \in$ $I$, of the linear operators in $X$. The uniqueness of $w$ implies that $w=u$ on $t \in\left[0, T_{0}\right]$. Hence $u$ is a unique classical solution of (1) and (11) and $u \in C\left(\left[0, T_{0}\right]: Y\right) \cap C^{1}\left(\left(0, T_{0}\right]: X\right)$. 


\section{Conclusion}

The present paper contains results concerning the existence and uniqueness of classical solutions for a quasilinear delay integrodifferential equation in Banach spaces. The result shows that the Banach fixed point theorem can effectively used to study the regularity of solutions for abstract quasilinear delay integrodifferential equation. Under suitable assumptions we have also proved the classical solutions for quasilinear integrodifferential equation with time varying delay and nonlocal condition.

Acknowledgement. The authors are thankful to the referee for the improvement of the paper.

\section{References}

[1] D. Bahuguna, Quasilinear integrodifferential equations in Banach spaces, Nonlinear Anal. 24 (1995), no. 2, 175-183.

[2] _ Regular solutions to quasilinear integrodifferential equations in Banach spaces, Appl. Anal. 62 (1996), no. 1-2, 1-9.

[3] K. Balachandran and D. G. Park, Existence of solutions of quasilinear integrodifferential evolution equations in Banach spaces, Bull. Korean Math. Soc. 46 (2009), no. 4, 691700 .

[4] K. Balachandran and F. Paul Samuel, Existence of solutions for quasilinear delay integrodifferential equations with nonlocal conditions, Electron. J. Differential Equations 2009 (2009), no. 6, 1-7.

[5] K. Balachandran and K. Uchiyama, Existence of local solutions of quasilinear integrodifferential equations in Banach spaces, Appl. Anal. 76 (2000), no. 1-2, 1-8.

[6] - Existence of solutions of quasilinear integrodifferential equations with nonlocal condition, Tokyo J. Math. 23 (2000), no. 1, 203-210.

[7] L. Byszewski, Theorem about existence and uniqueness of continuous solution of nonlocal problem for nonlinear hyperbolic equation, Appl. Anal. 40 (1991), no. 2-3, 173-180.

[8] _ Uniqueness criterion for solution of abstract nonlocal Cauchy problem, J. Appl. Math. Stochastic Anal. 6 (1993), no. 1, 49-54.

[9] - Theorems about the existence and uniqueness of solutions of a semilinear evolution nonlocal Cauchy problem, J. Math. Anal. Appl. 162 (1991), no. 2, 494-505.

[10] M. G. Crandall and P. E. Souganidis, Convergence of difference approximations of quasilinear evolution equations, Nonlinear Anal. 10 (1986), no. 5, 425-445.

[11] K. Furuya, Analyticity of solutions of quasilinear evolution equations, Osaka J. Math. 18 (1981), no. 3, 669-698; Analyticity of solutions of quasilinear evolution equations. II, Osaka J. Math. 20 (1983), no. 1, 217-236.

[12] M. Kanagaraj and K. Balachandran, Existence of solutions of quasilinear integrodifferential equations in Banach spaces, Far East J. Math. Sci. 4 (2002), no. 3, 337-350.

[13] S. Kato, Nonhomogeneous quasilinear evolution equations in Banach spaces, Nonlinear Anal. 9 (1985), no. 10, 1061-1071.

[14] T. Kato, Quasi-linear equations of evolution, with applications to partial differential equations, Spectral theory and differential equations (Proc. Sympos., Dundee, 1974; dedicated to Konrad Jorgens), pp. 25-70. Lecture Notes in Math., Vol. 448, Springer, Berlin, 1975.

[15] _ Abstract evolution equations, linear and quasilinear, revisited, Functional analysis and related topics, 1991 (Kyoto), 103-125, Lecture Notes in Math., 1540, Springer, Berlin, 1993. 
[16] A. Lunardi, Global solutions of abstract quasilinear parabolic equations, J. Differential Equations 58 (1985), no. 2, 228-242.

[17] M. G. Murphy, Quasilinear evolution equations in Banach spaces, Trans. Amer. Math. Soc. 259 (1980), no. 2, 547-557.

[18] H. Oka, Abstract quasilinear Volterra integrodifferential equations, Nonlinear Anal. 28 (1997), no. 6, 1019-1045.

[19] H. Oka and N. Tanaka, Abstract quasilinear integrodifferential equations of hyperbolic type, Nonlinear Anal. 29 (1997), no. 8, 903-925.

[20] A. Pazy, Semigroups of Linear Operators and Applications to Partial DifferentialEquations, Springer, New York, 1983.

[21] A. Yagi, Abstract quasilinear evolution equations of parabolic type in Banach spaces, Boll. Un. Mat. Ital. B (7) 5 (1991), no. 2, 341-368.

DONG GUN PARK

Department of Mathematics

DONG-A UNIVERSITY

BusAn 604-714, KoreA

E-mail address: dgpark@dau.ac.kr

KRISHNAN BALACHANDRAN

Department of Mathematics

Bharathiar UNIVERSITY

Coimbatore 641046, India

E-mail address: kbkb1956@yahoo.com

Francis Paul Samuel

Department of Mathematics

Bharathiar UNIVERSITY

Coimbatore 641046, IndiA

E-mail address: paulsamuel_f@yahoo.com 